\title{
Delirium: An Acute Confusional State
}

\section{Shoukat M and Iqbal RK* \\ Institute of Molecular Biology and Biotechnology, Bahauddin Zakariya University, Pakistan}

*Corresponding author: Rana Khalid Iqbal, Institute of Molecular Biology and Biotechnology, Bahauddin Zakariya University, Multan-60800, Pakistan, Tel: +92-619210463; Email: khalid.iqbal@bzu.edu.pk

\section{Mini Review \\ Volume 2 Issue 1}

Received Date: May 22, 2019

Published Date: June 11, 2019

DOI: $10.23880 /$ aabsc- 16000132

\begin{abstract}
Delirium is a frequent ineffectiveness in brain functioning. It is a disturbance in attention and awareness that develops over a short period of time and it is a neurocognitive disorder. Delirium provides an idiosyncratic chance for a better understanding of the neurocognitive reserve. Acute illness considered as a basis of delirium and it is usually activated by head trauma and recent surgeries. Delirium like dementia is clearly remarkable by a general disability in which a condition of being unable to perform as a consequence of physical and mental unfitness. It has also been observed that delirium can increase the rate of development of dementia. Unfortunately, the development of delirium or acute brain dysfunction is considered a non-essential component of a critical or unfavorable disease. The agitation and inactivity that is occurred in a delirium can increase the risk or danger of complications. Delirium is a major cause and the problem of hospitalization and should be considered a medical crisis. For these reasons its identification, prescription, and effective treatment are essential. For delirium treatment, the most common antipsychotic drugs are used. Emerging pharmacological strategies which include various antipsychotics drugs for addressing delirium should be combined with non-pharmacological approaches which are designed as a first line interventions.
\end{abstract}

Keywords: Delirium; Mental Disorder; Cognitive State; Prevention; Dementia

\section{Introduction}

Delirium is a frequent clinical syndrome described by distraction and by the acute loss of cerebral function such as remembering, thinking and reasoning of sufficient severity to interfere with daily functioning. As the first century $\mathrm{AD}$ in which the mental disorders were defined which occurred through head trauma and fever, the word 'delirium' for the first time was used as a medical term. Over time, the term delirium has been extended gradually so that it can explain a reversible syndrome which is severe, temporary and fluctuating, and which takes place in the setting of a medical condition [1]. Previously in ancient times, the word delirium was used to categorize an agitated and confused person while lethargus (a period of inactivity or sleep-like state in some arthropods and nematodes) was used to characterize a quietly confused person Smith, et al. [2]. Delirium is a sign of acute neuropsychological disorder which is explained by the Diagnostic and Statistical Manual of Mental Disorders(DSM-5) as a state in which normal mental functioning is disrupted for a short period of time, 
fluctuates and is ushered by a cognition change which is a change in higher brain functions such as reasoning and memory Pandharipande, et al. [3]. In older people, it is a clinical syndrome that is capable of being prevented and also can be described as an acute disorder of mental action and attention which is common and deadly. More than2.6 million adults 65 years and older every year possess delirium and considered $\$ 164$ billion in annual health care expenditures in the United States. Delirium and dementia synchronized frequently. Moreover, distraction or lack of concentration that is predicted in delirium prone to occur in the later stages of dementia. Another idiosyncratic feature of delirium that is rare to dementia, psychosis or depression is the variation in the level of consciousness [4]. Delirium is related with increased morbidity(cognitive state), mortality and length of hospital stay furthermore an increased use of health care systems that provide health care services to populations in need and costs [5]. Cognitive failure the absent -mindedness holds within both delirium and dementia and amnesic disorders [6].

\section{Medical Strategies for Delirium}

Integrative approaches for the prevention and global management of delirium have been properly studied up till now for extremely unwell adults and children [2]. To minimize the development of delirium and its harmful results prevention is the most efficacious strategy an approximately $30-40 \%$ of cases of delirium are capable of being prevented [1].

\section{Pharmacological}

To overcome the symptoms of delirium rather than abolishing its main cause of occurrence pharmacological therapy is preferred. Besides In case of hyperactive delirium subtype pharmacological treatment strategy is safe. Generally, when the medication is suggested for the prevention of delirium it should be noted that whether vascular dementia without regard of age is an issue or not [7]. Haloperidol (Haldol) is a traditional antipsychotic that is used for the treatment of delirium more frequently [2]. After cardiac surgery, the conventional sedatives which have a sleep-inducing effect used for the care of patients include propofol, morphine, and midazolam. While getting dexmedetomidine patients can be safely detubated and remain asleep and on getting analgesia they suddenly breathe. For the treatment of delirium, the most commonly used antipsychotic drugs are haloperidol, olanzapine, and risperidone [8].

\section{Non-Pharmacological}

Non-pharmacological therapies designed as first-line interventions for the prevention and medical management of delirium (postoperative) according to the recommendation of clinical practice guidelines recently published [8]. Schieveld and coworkers for critically ill children with delirium initiated an intervention strategy which includes the parental presence, participation of child psychiatrists, well-known music and pictures of frequent people. For a delirium treatment and determent the aim of a multicomponent approach based on reducing predisposing factors [2].

\section{Conclusion}

Delirium is a neuropsychological disorder of the brain. It is an acute, transient and fluctuating disturbance in cognition and consciousness level. Delirium is the most common and damaging disorder. To avoid the harmful effects of delirium it should be treated through medical strategies.

\section{References}

1. Manuscript A (2011) Delirium in elderly adults: diagnosis, prevention and treatment. Nat Rev Neurol 5(4): 210-220.

2. Smith HAB, Fuchs DC, Pandharipande PP, Barr FE, Ely EW (2010) Delirium: An Emerging Frontier in the Management of Critically Ill Children. Ctitical Care Clinic 25(3): 593-614.

3. Pandharipande P, Wesley EE, Rakesh CA, Michele CB, Malaz AB, et al. (2018) The intensive care delirium research agenda: a multinational, interprofessional perspective. Intensive Care Med 43(9): 1329-1339.

4. Oh ES, Fong TG, Hshieh TT, Inouye K, Istrael B, et al. (2018) Delirium in Older Persons Advances in Diagnosis and Treatment. JAMA 318(12): 1161-1174.

5. Abraha I, Trotta F, Rimland JM, Cruz-jentoft A, Lattanzio F, et al. (2015) Efficacy of NonPharmacological Interventions to Prevent and Treat Delirium in Older Patients: A Systematic Overview. The SENATOR project ONTOP Series. Plos One 10(6): e0123090.

6. Keeley P (2009) Supportive and palliative care Search date February 2009 Supportive and palliative care. 


\section{Annals of Advanced Biomedical Sciences}

7. Arbabi M, Shahhatami F, Mojtahedzadeh M, Mohammadi M (2018) Adaptation of the Pharmacological Management of Delirium in ICU Patients in Iran : Introduction and Definition. Iran J Psychiatry 13(1): 65-79.
8. Evans AS, Weiner MM, Arora RC, Chung I, Deshpande $\mathrm{R}$, et al. (2016) Current approach to diagnosis and treatment of delirium after cardiac surgery. Ann Card Anaesth 19(2): 328-337. 